PROCEEDINGS OF THE

AMERICAN MATHEMATICAL SOCIETY

Volume 125, Number 7, July 1997, Pages 2175-2177

S 0002-9939(97)03929-4

\title{
BASIC DIFFERENTIAL FORMS FOR ACTIONS OF LIE GROUPS. II
}

\author{
PETER W. MICHOR
}

(Communicated by Roe W. Goodman)

\begin{abstract}
The assumption in the main result of Basic differential forms for actions of Lie groups (Proc. Amer. Math. Soc. 124 (1996), 1633-1642) is removed.
\end{abstract}

Let $G$ be a Lie group which acts isometrically on a Riemannian manifold $M$. A section of the Riemannian $G$-manifold $M$ is a closed submanifold $\Sigma$ which meets each orbit orthogonally. In this situation the trace on $\Sigma$ of the $G$-action is a discrete group action by the generalized Weyl group $W(\Sigma)=N_{G}(\Sigma) / Z_{G}(\Sigma)$, where $N_{G}(\Sigma):=\{g \in G: g . \Sigma=\Sigma\}$ and $Z_{G}(\Sigma):=\{g \in G: g . s=s$ for all $s \in \Sigma\}$. A differential form $\varphi \in \Omega^{p}(M)$ is called $G$-invariant if $g^{*} \varphi=\varphi$ for all $g \in G$ and horizontal if $\varphi$ kills each vector tangent to a $G$-orbit. We denote by $\Omega_{\text {hor }}^{p}(M)^{G}$ the space of all horizontal $G$-invariant $p$-forms on $M$ which are also called basic forms.

In the paper [2] it was shown that for a proper isometric action of a Lie group $G$ on a smooth Riemannian manifold $M$ admitting a section $\Sigma$ the restriction of differential forms induces an isomorphism

$$
\Omega_{\mathrm{hor}}^{p}(M)^{G} \stackrel{\cong}{\rightrightarrows} \Omega^{p}(\Sigma)^{W(\Sigma)}
$$

between the space of horizontal $G$-invariant differential forms on $M$ and the space of all differential forms on $\Sigma$ which are invariant under the action of the generalized Weyl group $W(\Sigma)$ of the section $\Sigma$, under the following assumption:

For each $x \in \Sigma$ the slice representation $G_{x} \rightarrow O\left(T_{x}(G . x)^{\perp}\right)$ has a generalized Weyl group which is a reflection group.

In this paper we will show that this result holds in general, without any assumption. Notation is as in [2], which is used throughout. For more information on $G$-manifolds with sections see the seminal paper [3].

1. Polar representations. Let $G$ be a compact Lie group and let $\rho: G \rightarrow G L(V)$ be an orthogonal representation in a finite dimensional real vector space $V$ which admits a section $\Sigma$. Then the section turns out to be a linear subspace and the representation is called a polar representation, following Dadok [1], who gave a complete classification of all polar representations of connected Lie groups.

Received by the editors January 31, 1996.

1991 Mathematics Subject Classification. Primary 57S15, 20 F55.

Key words and phrases. Orbits, sections, basic differential forms.

Supported by Project P 10037-PHY of 'Fonds zur Förderung der wissenschaftlichen Forschung'.

(C)1997 American Mathematical Society 
Theorem. Let $\rho: G \rightarrow O(V)$ be a polar orthogonal representation of a compact Lie group $G$, with section $\Sigma$ and generalized Weyl group $W=W(\Sigma)$. Let $B \subset V$ be an open ball centered at 0 .

Then the restriction of differential forms induces an isomorphism

$$
\Omega_{h o r}^{p}(B)^{G} \stackrel{\cong}{\cong} \Omega^{p}(\Sigma \cap B)^{W(\Sigma)} .
$$

Proof. We only treat the case $B=V$. The restriction to an open ball can be proved as in [2], 3.8. Let $i: \Sigma \rightarrow V$ be the embedding. It is easy to see (and proved in $[2], 2.4)$ that the restriction $i^{*}: \Omega_{\text {hor }}^{p}(V)^{G} \rightarrow \Omega^{p}(\Sigma)^{W(G)}$ is injective, so it remains to prove surjectivity. Let $G_{0}$ be the connected component of $G$. From [1], lemma 1 one concludes:

A subspace $\Sigma$ of $V$ is a section for $G$ if and only if it is a section for $G_{0}$. Thus $\rho$ is a polar representation for $G$ if and only if it is a polar representation for $G_{0}$.

The generalized Weyl groups of $\Sigma$ with respect to $G$ and to $G_{0}$ are related by

$$
W\left(G_{0}\right)=N_{G_{0}}(\Sigma) / Z_{G_{0}}(\Sigma) \subset W(G)=N_{G}(\Sigma) / Z_{G}(\Sigma),
$$

since $Z_{G}(\Sigma) \cap N_{G_{0}}(\Sigma)=Z_{G_{0}}(\Sigma)$.

Let $\omega \in \Omega^{p}(\Sigma)^{W(G)} \subset \Omega^{p}(\Sigma)^{W\left(G_{0}\right)}$. Since $G_{0}$ is connected the generalized Weyl group $W\left(G_{0}\right)$ is generated by reflections (a Coxeter group) by [1], remark after proposition 6 . Thus we may apply [2], theorem 3.7, which asserts that then

$$
i^{*}: \Omega_{\text {hor }}^{p}(V)^{G_{0}} \stackrel{\cong}{\cong} \Omega^{p}(\Sigma)^{W\left(G_{0}\right)}
$$

is an isomorphism, and we get $\varphi \in \Omega_{\text {hor }}^{p}(V)^{G_{0}}$ with $i^{*} \varphi=\omega$. Let us consider

$$
\psi:=\int_{G} g^{*} \varphi d g \in \Omega_{\mathrm{hor}}^{p}(V)^{G},
$$

where $d g$ denotes Haar measure on $G$. In order to show that $i^{*} \psi=\omega$ it suffices to check that $i^{*} g^{*} \varphi=\omega$ for each $g \in G$. Now $g(\Sigma)$ is again a section of $G$, thus also of $G_{0}$. Since any two sections are related by an element of the group, there exists $h \in G_{0}$ such that $h g(\Sigma)=\Sigma$. Then $h g \in N_{G}(\Sigma)$ and we denote by $[h g]$ the coset in $W(G)$, and we may compute as follows:

$$
\begin{aligned}
\left(i^{*} g^{*} \varphi\right)_{x} & =\left(g^{*} \varphi\right)_{x} \cdot \Lambda^{p} T i=\varphi_{g(x)} \cdot \Lambda^{p} T g \cdot \Lambda^{p} T i \\
& =\left(h^{*} \varphi\right)_{g(x)} \cdot \Lambda^{p} T g \cdot \Lambda^{p} T i, \quad \text { since } \varphi \in \Omega_{\mathrm{hor}}^{p}(M)^{G_{0}} \\
& =\varphi_{h g(x)} \cdot \Lambda^{p} T(h g) \cdot \Lambda^{p} T i=\varphi_{i[h g](x)} \cdot \Lambda^{p} T i \cdot \Lambda^{p} T([h g]) \\
& =\left(i^{*} \varphi\right)_{[h g](x)} \cdot \Lambda^{p} T([h g]) \\
& =\omega_{[h g](x)} \cdot \Lambda^{p} T([h g])=[h g]^{*} \omega=\omega .
\end{aligned}
$$

2. Theorem. Let $M \times G \rightarrow M$ be a proper isometric right action of a Lie group $G$ on a smooth Riemannian manifold $M$, which admits a section $\Sigma$.

Then the restriction of differential forms induces an isomorphism

$$
\Omega_{h o r}^{p}(M)^{G} \stackrel{\cong}{\cong} \Omega^{p}(\Sigma)^{W(\Sigma)}
$$

between the space of horizontal G-invariant differential forms on $M$ and the space of all differential forms on $\Sigma$ which are invariant under the action of the generalized Weyl group $W(\Sigma)$ of the section $\Sigma$. 
This is the Main Theorem 2.4 of [2], without the assumption made there.

Proof. Injectivity is proved in [2], 2.4, without using the assumption. Surjectivity can be proved as in [2], section 4 , where one replaces the use of [2], 3.8, by Theorem 1 above.

\section{REFERENCES}

1. Dadok, J., Polar coordinates induced by actions of compact Lie groups, TAMS 288 (1985), 125-137. MR 86k:22019

2. Michor, Peter W., Basic differential forms for actions of Lie groups, Proc. AMS 124 (1996), 1633-1642. MR 96g:57041

3. Palais, R. S.; Terng, C. L., A general theory of canonical forms, Trans. AMS 300 (1987), 771-789. MR 88f:57069

Institut für Mathematik, Universität Wien, Strudlhofgasse 4, A-1090 Wien, Austria

E-mail address: Peter.Michor@univie.ac.at 